\title{
Nucleating Acoustic Cavitation with Optically Heated Nanoparticles
}

Cite as: AIP Conference Proceedings 838, 225 (2006); https:// doi.org/10.1063/1.2210350

Published Online: 12 June 2006

R. A. Roy, C. H. Farny, T. Wu, R. G. Holt, and T. W. Murray

ARTICLES YOU MAY BE INTERESTED IN

A Theoretical Study of Gas Bubble Dynamics in Tissue AIP Conference Proceedings 838, 217 (2006); https:// doi.org/10.1063/1.2210349

Thermal Lesion Development in Bubble-Mediated HIFU: Modeling AIP Conference Proceedings 829, 333 (2006); https:// doi.org/10.1063/1.2205492

\section{Conference Proceedings}

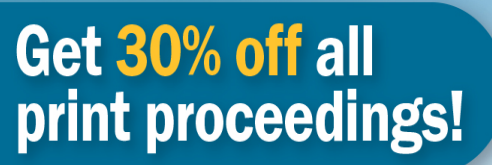

Enter Promotion Code PDF30 at checkout 


\title{
Nucleating Acoustic Cavitation with Optically Heated Nanoparticles
}

\author{
R. A. Roy, C. H. Farny, T. Wu, R. G. Holt, T. W. Murray \\ Department of Aerospace and Mechanical Engineering, Boston University, Boston, MA 02215
}

\begin{abstract}
The utilization of cavitation in high intensity focused ultrasound (HIFU) therapy requires the presence of nucleation sites; nucleation threshold pressures in tissues can exceed 4-5 $\mathrm{MPa}$. We investigate the efficacy of transient vapor cavity generation from laser-illuminated gold nanoparticles as a means for nucleating cavitation. An acrylamide tissue phantom seeded with 82-nm diameter gold particle was exposed to $5 \mathrm{~ns}$ pulses from a $532 \mathrm{~nm} \mathrm{Nd}$ :Yag laser. Acoustic emissions from inertial cavitation were detected by a $15 \mathrm{MHz}$ broadband focused transducer at a laser energy of $0.10 \mathrm{~mJ} /$ pulse and a HIFU peak-negative focal pressure as low as $0.92 \mathrm{MPa}$. In comparison, a peak-negative focal pressure of 4.50 MPa was required to nucleate detectable cavitation without laser illumination. A simple analysis reveals that vapor cavities are formed that grow to the Blake radius, at which point they undergo rapid expansion and collapse.
\end{abstract}

Keywords: cavitation, nucleation, laser, nanoparticles.

PACS: 43.35.Ei, 43.80.Gx, 43.80.Sh

\section{INTRODUCTION}

Cavitation is implicated in a number of mechanical and thermal processes relevant to therapeutic ultrasound in general, and high-intensity focused ultrasound (HIFU) therapy in particular; see [1] for a comprehensive discussion. Bubbly media can alter the dispersive and dissipative characteristics in the propagation path, leading to defocusing effects and accelerated tissue heating. Stable cavitation generates microsteaming flows that can disrupt cells and promote mass transfer by breaking down boundary layers. Inertial cavitation promotes collapse microjets, radiated shock waves, chemical reactions, and enhanced localized tissue heating due to the rapid absorption of broadband acoustic emissions [1,2]. In order to effectively exploit these effects, practitioners must establish the cavitation field at the lowest possible acoustic pressures, however, nucleation thresholds for many tissues can be quite high, ranging from 3-4 $\mathrm{MPa}$ in blood to over $6 \mathrm{MPa}$ in muscle tissue. Ultrasound contrast agents have been employed to promote cavitation [3,4], however, UCAs are short lived due diffusional instability and/or mechanical disruption by the HIFU acoustic field.

Below we describe an in vitro investigation of an alternate approach to the cavitation nucleation problem. The technique is inspired by prior work on optoacoustic contrast enhancement using gold nanoparticles conjugated to antibodies bound to the surface of breast cancer cells implanted at 5-6-cm depths in vitro [5]. These particles preferentially absorb laser light, yielding transient vapor cavities and subsequent acoustic emissions upon collapse. Such cavities, when exposed to tensile 
acoustic stress, will also serve as nucleation sites for acoustic cavitation. The primary advantages of this approach are (1) the moment of nucleation can be precisely timed to coincide with the peak rarefaction pressure phase and (2) the particles are durable and can be "reactivated" over an over again, thereby providing nuclei "on demand."

\section{DESCRIPTION OF THE EXPERIMENT}

We investigate this concept using 82-nm-diameter gold particles embedded in a aqueous, non-degassed, transparent, polyacrylamide gel phantom at a concentration of $10^{9}$ particles per $\mathrm{ml}$. The phantom was submerged in a tank of degassed water and exposed to individual $5 \mathrm{nsec}$ pulses from a $0.8-\mathrm{mm}$ diameter beam of $532-\mathrm{nm}$ light generated by a frequency-doubled Nd:YAG laser. A HIFU source operating at 1.1 $\mathrm{MHz}$ launched 10-cycle bursts in the form of a focused beam with a focal length of approximately $6 \mathrm{~cm}$. The pulse repetition frequency for the HIFU exposure was $0.1 \mathrm{~Hz}$ and the acoustic and optical beams were aligned collinear. To trigger the laser, we electronically delayed the HIFU drive to ensure that the laser fired at the same instant that the acoustic pulse traversed the focus. This delay could be precisely varied to explore the relationship between the onset of nucleation and the phase of the acoustic field in the cavitation sensing volume (described below). Detailed descriptions of the sample preparation, exposure geometry, and the HIU and laser generation systems are presented [6], and are not reproduced here.

To detect inertial cavitation activity, we employed a passive cavitation detector (PCD) consisting of a $15 \mathrm{MHz}$ broad-band focused receiving transducer positioned confocal to the HIFU transducer. The detection axis was perpendicular to the HIFU beam and the detection beamwidth of approximately $0.6 \mathrm{~mm}$ in the focal zone. The overlapping confocal region defined the cavitation detection zone. The received signal was linearly amplified (40 dB) and high-pass filtered above $5 \mathrm{MHz}$ (passive, 4-stage Butterworth). Thus configured, the PCD was sensitive to broad-band acoustic emissions from inertial cavitation collapses. Single-shot PCD signals were digitized (8-bit, $50 \mathrm{Msample/sec)} \mathrm{and} \mathrm{stored} \mathrm{on} \mathrm{computer} \mathrm{memory} \mathrm{for} \mathrm{processing.} \mathrm{See} \mathrm{[6]} \mathrm{for} \mathrm{a}$ detailed description of cavitation detection instrumentation.

We applied a time gate to the PCD data to eliminate laser RF noise and then converted to the frequency domain by way of the FFT. The quantified indicator of cavitation activity is the emission level (EL) defined as follows:

$$
E L=\int_{5 M H z}^{25 M H z}|X(f)|^{2} d f
$$

where the integrand is the square of the magnitude spectrum. This measure of the broadband noise power emanating from the cavitation detection zone serves as an approximate measure of inertial cavitation activity. An additional objective parameter indicative of activity is termed the emission gain (EG) defined by:

$$
E G=10 \log \left[\frac{\langle E L\rangle}{\left\langle E L_{\text {background }}\right\rangle}\right]
$$


where the background emission level is measured with HIFU on and the laser beam blocked, and the averages are computed over 10 observations. The EG is an indicator of the extent to which bubble-collapse emissions exceed the background noise, and accounts for all systemic noise sources.

\section{RESULTS AND DISCUSSION}

As a first step, we measured conditions required to generate detectable cavitation activity from the laser alone and from the HIFU alone; particles were present in both cases. The minimum laser energy required to form detectable microcavitation was 4 $\mathrm{mJ}$. The minimum HIFU peak negative pressure required to produce detectable microcavitation was $4.50 \mathrm{MPa}$. This is the nucleation threshold pressure for the phantom material.

Several experiments were run that demonstrated the synergistic behavior of light and sound for nucleating cavitation. We observed a suppression of nucleation threshold pressures for incident laser energies as low as $0.09 \mathrm{~mJ}$, which is a factor of 45 less than the energy required to nucleate detectable vapor bubble collapses in the absence of acoustic forcing. At the maximum laser energy employed $(0.11 \mathrm{~mJ})$, the nucleation threshold pressure was somewhere between 0.8 and $0.9 \mathrm{MPa}$, a greater than fourfold reduction in the nucleation threshold. The suite of results is too extensive to present here; the reader is referred to [6] for a detailed report.

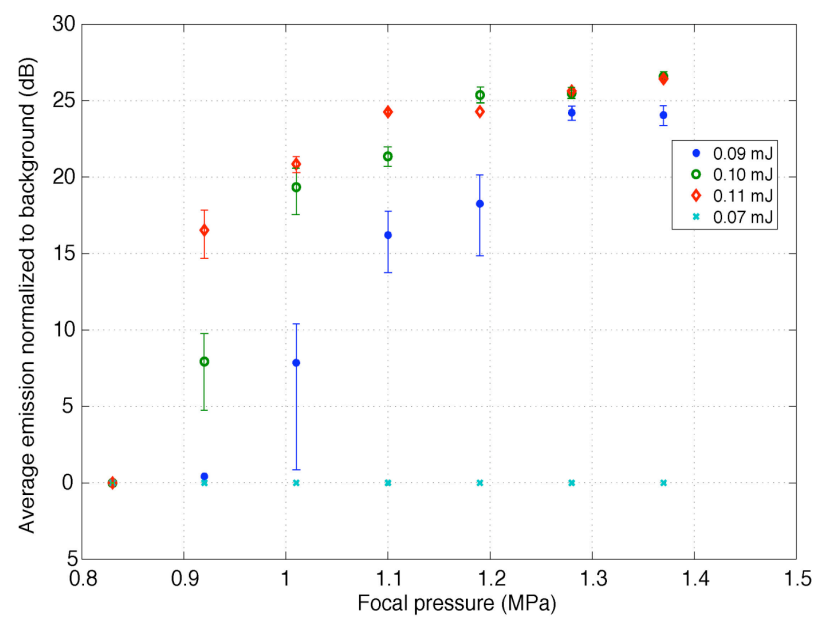

FIGURE 1. Inertial cavitation emissions relative to background as a function of laser energy and peak negative HIFU pressure. The errors bars are based on the standard error for a 10-point sample.

Figure 1 provides a summary view of the role of light and sound in suppressing nucleation thresholds in the presence of gold nanoparticles. The variable delay was set to fire the laser beam $0.15 \mu \mathrm{s}$ after the fifth acoustic cycle arrived at the HIFU focal plane. Below a laser energy of about $0.08 \mathrm{~mJ}$, no cavitation is observed; this is the illumination threshold energy for the range of acoustic pressures employed. For light exposures at and above $0.08 \mathrm{~mJ}$, a clearly demarcated inertial cavitation threshold 
pressure exists, and this threshold is reduced as the laser energy is increased. It is important to note that both the laser energy and acoustic pressure thresholds are significantly lower than those measured with light and sound alone. The results summarized in Fig. 1 serve to prove the concept.

It is instructive to consider the physics that govern this process. In order to nucleate inertial cavitation, the vapor cavity produced by particle heating must grow from an initial radius of approximately $41 \mathrm{~nm}$ to a size equal to or greater than the Blake radius [ref 1, Sect. 4.3.1] for the acoustic pressure used. Is this, in fact, the case for the data given in Fig. 1? The energy absorbed by a particle is given approximately by:

$$
Q=E_{L} \beta\left[\frac{R_{s}}{R_{L}}\right]^{2}
$$

where $E_{L}$ is the energy in the laser beam, $\beta$ is absorption efficiency $(\approx 3.3), R_{S}$ is the radius of the scatterer $(41 \mathrm{~nm})$ and $R_{L}$ is the radius of the laser bean $(0.8 \mathrm{~mm})$. We assume that all this energy is absorbed as heat and forms a thin vapor shell surrounding the particle. The mass of vapor in this shell, $m$, is given by:

$$
Q=m[C \Delta T+h] ; \Delta T=T_{c}-T_{o}
$$

where $C$ is the heat capacity of the material and $h$ is the heat of vaporization (both assumed to be that of water) $T_{o}$ is the ambient temperature and $T_{c}$ is the critical temperature $(520 \mathrm{~K})$. In specifying the critical temperature, we account for the pressure jump at the interface due to surface tension (3.5 MPa for a 41-nm radius cavity). Once we know the mass and temperature of the vapor produced, we go to the steam tables to determine the corresponding specific volume, from which we can compute the maximum size of the cavity formed in the phantom.

To test this simple model, consider the fact that the threshold pressure for $0.01 \mathrm{~mJ}$ exposure was about $0.87 \mathrm{MPa}$. The resulting values for the maximum cavity size and the Blake radius at this pressure are $105 \mathrm{~nm}$ and $75 \mathrm{~nm}$, respectively. (In this calculation, the Blake radius is corrected to account for the presence of the particle, which is assumed to be rigid). Note that the nuclei size does in, fact, exceed the Blake size, as it must. The fact that the two sizes are similar is consistent with the notion that we are measuring a threshold condition. [Work supported by the Dept. of the Army (award No. DAMD17-02-2-0014) and the Center for Subsurface Sensing and Imaging Systems (NSF ERC Award No. EEC-9986821).]

\section{REFERENCES}

1. Leighton, T. G., The Acoustic Bubble, Academic, London, 1997.

2. Holt, R. G., and Roy, R. A., Ultrasound Med. Biol. 27, 1399-1412 (2001).

3. Tran,B. C., Seop, J., Hall, T. L., Fowlkes, J. B., and Cain, C. A., IEEE Trans. Ultrason. Ferroelectr. Freq. Control 50, 1296-1304 (2003).

4. Sassaroli, E., and Hynynen, K., J. Acoust. Soc. Am. 115, 3225-3243 (2004).

5. Eghtedari, M., Motamedi, M., Popov, V. L., Kotov, N. A., and Oraevsky, A. A., Photons Plus Ultrasound: Imaging and Sensing, Proceedings of SPIE 5320, pp. 365-377 (2004).

6. Farny, C. H., Wu, T., Holt, R. G., Murray, T. W., and Roy, R. A., Acoust. Res. Lett. Online 6, 138143 (2005). 